\title{
Multidetector Computed Tomographic Evaluation of Spontaneous Intracranial Haemorrhage
}

\author{
Mini.M.V ${ }^{1}$, Brahamadathan.M.N $^{2}$,Shaji Abraham ${ }^{3}$ \\ '(Department Of Radiodiagnosis, Government Medical College, Thrissur, India) \\ ${ }^{2}$ (Department Of Radiodiagnosis, Government Medical College, Thrissur, India) \\ ${ }^{3}$ (Department Of Neurology, Government Medical College, Thrissur, India)
}

\begin{abstract}
Spontaneous intracranial hemorrhage is one of the major causes of acute cerebrovascular accidents. Hemorrhagic stroke accounts for approximately $15 \%$ of all stroke and is classified according to anatomic compartmentalization as intracerebral hemorrhage (ICH) (approximately two thirds) or subarachnoid hemorrhage ( $S A H)$ (approximately one third).It is very important to detect intracranial hemorrhage as early as possible for proper management as it may lead to rapid neurological deterioration and even death. Hence it is an indication for urgent neuro imaging. The aim of this work was to evaluate the computed tomography (CT) findings of spontaneous intracranial haemorrhage and to correlate the findings with 30 days clinical outcome.

Materials and methods -Study design was descriptive cross sectional study conducted in Department of Radiodiagnosis, Government Medical College Thrissur. All patients above one year of age with CT features of non traumatic intracranial hemorrhage were included in the study. Patients below one year, patients with head injury, patients with intra tumoral bleed and patients on thrombolytic therapy / on anticoagulants and ant platelet drugs were excluded. Study period was 20 months (April 2011 to November 2012). Sample size was 100. Method of collection of data - 100 consecutive patients with CT features of intracranial hemorrhage with no history of trauma are included in the study and followed up for 30 days. Outcome was studied prospectively based on Glasgow Outcome Scale as described for head injuries .Conclusion - The study concluded that intraparenchymal hemorrhage was the commonest variety of non traumatic spontaneous intracranial hemorrhage and basal ganglia was the commonest location. The significant predictors of 30 days mortality were intraventricular hemorrhage, midline shift, sub arachnoid extension of bleed, low GCS on admission $(\leq 8)$ and volume of hematoma $30 \mathrm{ml}$ and above.
\end{abstract}

Keywords: Computed tomography, intraparenchymal hemorrhage, subarachnoid hemorrhage

\section{Introduction}

Spontaneous brain hemorrhage is defined as bleeding into the brain substance by any cause that is of non traumatic origin.They are classified according to their anatomic location into intraparenchymal, subarachnoid, subdural and epidural hematomas. The worldwide incidence of intracerebral hemorrhage ranges from 10 to 20 cases per 100,000 population and increases with age ${ }^{1}$. Bleedings into the brain parenchyma and also hemorrhages into the subarachnoid space occur mostly spontaneously. Brain hematomas account for $10 \%$ to $12 \%$ and subarachnoid hemorrhages for $5 \%$ to $7 \%$ of all cases of stroke ${ }^{1}$. Bleeding into the subdural and epidural spaces is mostly traumatic in origin ${ }^{1}$. Intracerebral hemorrhage is more common in men than women, particularly in those older than 55 years of age. Hypertension increases the risk of intracerebral hemorrhage, particularly in persons, who are not compliant with antihypertensive medication, or are 55 years of age or younger, or are smokers. ${ }^{2}$

Depending on the underlying cause intracerebral hemorrhage is classified as either primary or secondary. Primary intracerebral hemorrhage, accounting for 78 to 88 percent of cases, originates from the spontaneous rupture of small vessels damaged by chronic hypertension or amyloid angiopathy ${ }^{1}$. Secondary intracerebral hemorrhage occurs in a minority of patients in association with vascular abnormalities (such as arteriovenous malformations and aneurysms), tumors, or impaired coagulation. SAH, or subarachnoid hemorrhage, is bleeding into the subarachnoid space - the area between the arachnoid membrane and the pia mater surrounding the brain. This may occur spontaneously, usually from a ruptured cerebral aneurysm, or may result from head injury. SAH is a form of stroke and comprises 1-7\% of all strokes. ${ }^{3}$

Until the advent of computed tomography (CT) in 1972, the diagnosis of spontaneous brain hemorrhage could only be inferred from the angiographic findings. CT revolutionized the diagnosis of intracranial disease, which became the primary diagnostic tool in the evaluation of a patient presenting with an acute stroke. There is no clinical scoring system to differentiate intracerebral hemorrhage from ischemic stroke reliably. Therefore timely brain imaging is the key to recognize intracerebral hemorrhage. Computed tomography detects symptomatic intracerebral hemorrhage within minutes of onset of symptoms and up to one week thereafter. Thus CT helps to study the spectrum of intracranial hemorrhage in relation to the etiology and 
clinical presentation. Since the natural history of the disease is not well known, the determination of the prognostic factors would contribute in selecting the therapeutic options.

\subsection{Aim Of Study}

1. Evaluation of the type, location and volume of intracranial hemorrhage.

2. To detect the following associated features of intraparenchymal hemorrhage

a) Intraventricular extension b) Mass effect in the form of midline shift.

3. To correlate the C.T features of intraparenchymal hemorrhage with 30 days clinical outcome.

\section{Materials and methods}

Study design was descriptive cross sectional study conducted in Department of Radiodiagnosis Government Medical College Thrissur. All patients above one year of age with CT features of non traumatic intracranial hemorrhage were included in the study. Patients below one year, patients with head injury, patients with intra tumoral bleed and patients on thrombolytic therapy / on anticoagulants and ant platelet drugs were excluded. Study period was 20 months (April 2011 to November 2012).Sample size was 100. Method of collection of data - 100 consecutive patients with CT features of intracranial haemorrhage with no history of trauma are included in the study and they are followed up for 30 days. Outcome was studied prospectively based on Glasgow Outcome Scale as described for head injuries .Study protocol was submitted to Institutional research committee and Ethical committee with necessary approvals obtained. Data was collected from patients after getting an informed consent .The collected personal information was kept strictly confidential.

\subsection{Technique}

Serial axial CT sections of brain were obtained from foramen magnum level to vertex at $4.8 \mathrm{~mm}$ interval in Siemens Somatom Emotion $-16^{\mathrm{TM}}$. The volume of ICH (Intracerebral hemorrhage) was measured by using the freehand tracing tools in the region of interest module from the Analyze software. According to various literatures we selected the following $\mathrm{CT}$ features of intracerebral hemorrhage as those most likely to be relevant for predictiing 30 days clinical outcome. They were - volume of hematoma $30 \mathrm{ml}$ and above, location of hematoma (whether supra or infra tentorial), the presence of mid line shift of $>5 \mathrm{~mm}$, evidence of intraventricular extension of hemorrhage and sub arachnoid extension of bleed. We also included GCS at admission as a significant predictor of 30 days clinical outcome. Patient outcome was studied prospectively with a clinical follow-up for one month. The Glasgow Outcome Scale as described for head injuries was used. This scale classifies patients into one of five mutually exclusive categories defined as 1)good recovery (capable of returning to the former level of function even with a minor deficit), 2) moderately disabled (independent but objectively or subjectively disabled), 3) severely disabled (dependent on others for the activities of daily living but capable of social interaction), 4) vegetative state (no social or relational interaction) and 5) death. Good recovery and moderately disabled comes under satisfactory outcome. Death as well as severely disabled and vegetative state comes under unsatisfactory outcome.

\subsection{Data Analysis}

Univariate analysis was performed to correlate the patient's outcome and each potential prognostic factor using the chi-square and t-test. A value of $\mathrm{p}<0.05$ was considered to be significant.

\section{Results}

We studied a total of 100 patients with nontraumatic intra cranial hemorrhage. Out of which 76 patients had intraparenchymal hemorrhage and 24 patients had subarachnoid hemorrhage (SAH). The number of intraparenchymal hemorrhage (IPH) was approximately three times higher than subarachnoid hemorrhage. In this study, out of the 76 patients with intra parenchymal hemorrhage 60 patients (79\%) were hypertensive and $16(21 \%)$ were non hypertensive. Out of 24 patients with subarachnoid hemorrhage $18(75 \%)$ were hypertensive and $6(25 \%)$ were non hypertensive. IPH was found most in the 60-69 age groups. Age distribution for SAH was most in the 50-59 age groups. No primary IVH or SDH was seen in this study. In this study, of 100 patients with intracranial hemorrhage, $62 \%$ patients were male and remaining $38 \%$ were females. Out of 76 patients with IPH, 52 were males and 24 were females. And out of 24 patients with SAH, 10 were males and 14 were females.

The commonest location of IPH was basal ganglia 32( 42\%) followed by lobar 18(24\%),cerebellum $10(13 \%)$,thalamus $7(9 \%)$,thalamoganglia $5(7 \%)$ and brain stem $4(5 \%)$. In this study, of the 76 patients with intraparenchymal hemorrhage 58(76\%) patients showed non lobar location and 18 (24\%) patients showed lobar location of the bleed.

50 out of $76(65 \%)$ patients of intraparenchymal hemorrhage had volume of bleed in the range of 0 $29 \mathrm{ml}, 21(28 \%)$ had volume in the range of $30-59 \mathrm{ml}$ and $5(7 \%)$ had volume in the range of above $60 \mathrm{ml}$.The 
same is summarized in table 1 . Out of 76 patients of intraparenchymal hemorrhage 27 patients $(36 \%)$ had intraventricular extension and 20 patients $(26 \%)$ had subarachnoid extension of the bleed. Midline shift noted in 19 patients(25\%). Out of 76 patients with IPH 28(37\%) patients died, 12 patients were in category 1of Glasgow Outcome Scale and 25 were in category 2, 10 patients were in category 3 and 1 was in category 4, i.e. 37(49\%) showed satisfactory outcome and $39(51 \%)$ patients showed unsatisfactory outcome.In this study out of 27 patients with IVH, 20 patients $\operatorname{died}(74 \%)$ and $7(26 \%)$ were alive . The 7 patients who were alive were in category 3 (severely disabled) of Glasgow Outcome Scale. Out of 14 patients with infratentorial bleed 6 patients(43\%) died and $8(57 \%)$ were alive. Out of eight live patients, two patients showed good recovery( category-1), five were in category- 2 and one was severely disabled (category-3). Of the 62 patients with supratentorial bleed, 22 patients died (36\%). In this study, out of 19 patients with midline shift 12 patients died (63\%). Out of the 7 live patients 1 was in vegetative state ( category-4),5 were severely disabled ( category3 )and 1 patient showed good recovery ( category-1).

Out of 20 patients with SAH, 16(80\%) patients died (category-5). Of the remaining 4 live patients, 1 showed good recovery ( category-1), 1 was in category-2 and 2 were in category-3. In this study out of 26 patients who had IPH volume $30 \mathrm{ml}$ and above, 14 patients (54\%)died. Of the remaining 12(46\%) live patients, 2 patients showed good recovery (category-1), 3 were moderately disabled (category-2), 6 were in category-3. Out of 5 patients who had volume $>60 \mathrm{ml} 4$ died (Table 2). Based on Glasgow Coma Scale patients were classified into two group's .i.e. GCS $\leq 8$ and more than 8 . Out of 24 patients who had GCS $\leq 8$, eighteen patients died and six were alive. Out of 6 live patients 2 were in category-2 and 4 were in category-3. Out of 52 patients who had GCS >8, only 10 patients died, 42 were alive and 1 was in category-4. 24 patients presented with primary SAH out of which18 patients were hypertensive. Of the 24 patients, 10 were in GCS $<8,11$ patients in GCS 9-12 and 3 were in GCS above 13.Out of 24, $11(46 \%)$ died. All the 10 patients with GCS $<8$ died. Intraventricular extension of bleed was noted in 5 patients and out of this, 4 died.

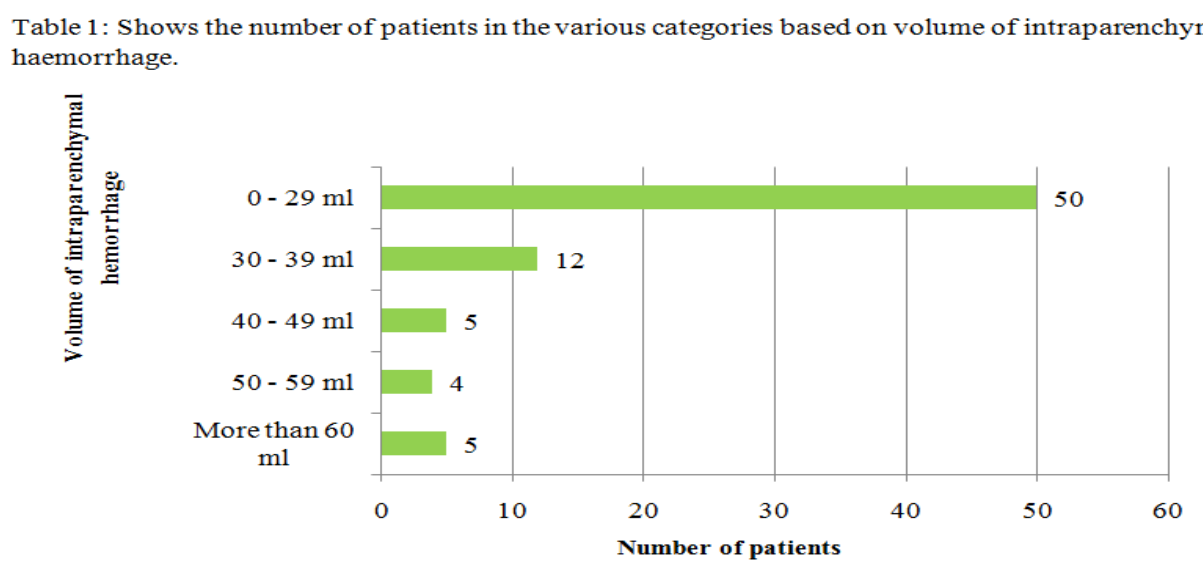

\section{Igures And Tables}

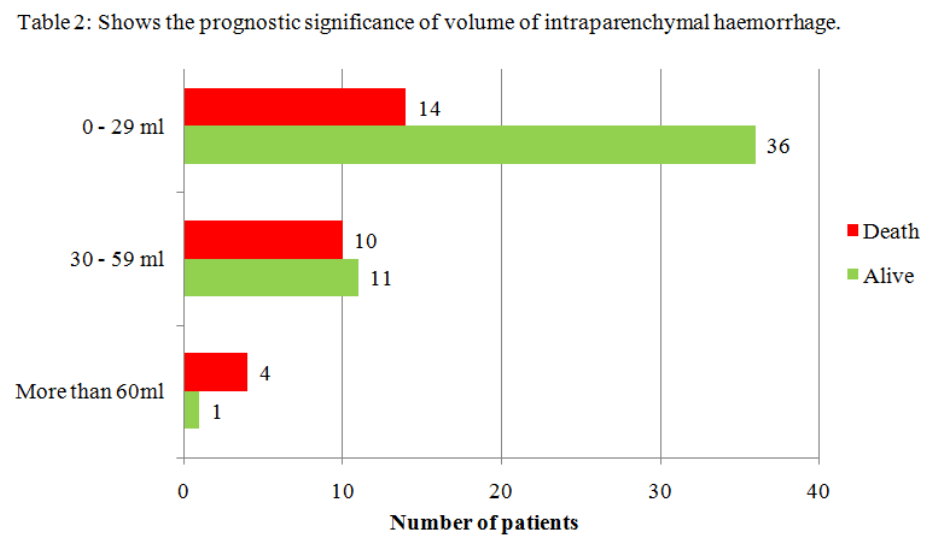

\section{Discussion}

In the study an analysis of 100 patients with non-traumatic intracranial haemorrhage was done . Based on socio-demographic and clinical profile of patients, study showed that the incidence of intracranial 
haemorrhage gradually increased with age and was most around 60years. Age distribution for IPH was maximum between 60-69 age groups and for SAH is maximum between 50-59 age groups. Males were noted to be the more affected population than the females with a frequency of $62 \%$ for males and $38 \%$ for females. Following radiological parameters of intracranial haemorrhage were evaluated:

1. Type and location of intracranial haemorrhage.

2. Volume of intracranial haemorrhage.

3. Intraventricular extension of hemorrhage.

4. Subarachnoid extension of hemorrhage.

5. Mass effect in the form of midline shift.

6. GCS at admission was also studied

In this study of 100 patients of spontaneous ICH, 76 patients had intraparenchymal hemorrhage (76\%) and 24 patients had SAH (24\%). In this study out of 76 patients with intra parenchymal hemorrhage 60 patients (79\%) were hypertensive and 16(21\%) were non hypertension. Out of 24 patients with subarachnoid hemorrhage $18(75 \%)$ were hypertensive and $6(25 \%)$ were non hypertensive.

In this study, supratentorial location of the bleed $(82 \%)$ was more common than infratentorial location $(18 \%)$. It was also shown that non lobar hemorrhage $(76.3 \%)$ was more common than lobar hemorrhage $(23.7 \%)$.

In our study, out of 76 patients with intraparenchymal hemorrhage, 32 patients had basal ganglia bleed $(42 \%)$ followed by 18 cases of lobar bleed, (23.7\%), 7 cases of thalamic bleed $(9.2 \%), 5$ patients had thalamo ganglia bleed (6.5\%), 10 patients had cerebellar bleed (13\%), and 4 had brainstem bleed (5\%). According to Justine Elliott, fifty percent of hypertension-related hemorrhage occurs in deep structures (basal ganglia and thalamus) and 30\% in superficial (lobar) areas ${ }^{4}$.According to Azra Zafar, basal ganglia (55\%) was the commonest site of bleed followed by thalamus. ${ }^{5}$ In another study by Kase C.S. et al showed that the location of hypertensive intracerebral hemorrhage was putaminal $33 \%$, lobar $23 \%$, thalamic $20 \%$, cerebellar $8 \%$, pontine $7 \%$ and miscellaneous $9 \%{ }^{6}$, which is matching with our study. According to RM Yousuf the commonest location of ICH was lobar (43.8\%) followed by basal ganglia / internal capsule $(28.1 \%)$ and multilobar $(13.1 \%){ }^{7}$ According to Lt Col MN Swamy majority (45\%) of the clots were lobar followed by basal ganglia bleed (35\%) ${ }^{8}$

This study correlated with three of the above mentioned studies which showed that commonest location of intracerebral hemorrhage was basal ganglia. Disparity with two studies mentioned above may be because more hypertensive patients were included in our study.

In this study out of 76 patients with IPH 28(37\%) patients died, out of live patients 12 patients were in category 1of Glasgow Outcome Scale and 25 were in category 2, 10 patients were in category 3 and 1 was in category4, i.e. 37(49\%) showed satisfactory outcome and 39(51\%) patients showed unsatisfactory outcome .Mortality rate was $37 \%$ in this study. Douglas and Haerer reported a mortality rate of $40 \%{ }^{9}$ and Dixon et al in 1985 reported $33 \%$ mortality ${ }^{10}$. According to RM Yousuf, the overall mortality rate was $32.5 \%$ and P. Daverat, M D reported mortality rate during the first month following ICH as $31^{6}$. The mortality rates in the above mentioned studies are comparable to our study.

In this study of 76 patients of intraparenchymal hemorrhage, 27 patients $(36 \%)$ had intraventricular extension. Out of this 27 patients with intraventricular extension, 20(74\%) patients died and 7(26\%) were alive. The 7 patients who were alive are in category 3 (severely disabled) of Glasgow Outcome Scale. The p-value was 0.000 (significant). That means there was significant association of intraventricular extension of IPH with high mortality and morbidity.

We have classified the patients with intraparenchymal hemorrhage according to the volume of haemorrhage. In our study 50 ( $65 \%$ ) patients had the volume of hematoma $<30 \mathrm{ml}$ and the remaining $26(35 \%)$ had volume 30\&above. Out of the 26 who had volume 30\&above, 14 patients died .Of the remaining 12 live patients 1 was in ( category-4), 6 were in( category-3), 3 were moderately disabled( category-2) and 2 patients showed good recovery( category-1). P value-0.03( significant). That means the majority of patients with larger bleed either died or survived with significant handicap.

Mass effect in the form of midline shift noted in 19 (23\%) cases of intraparenchymal hemorrhage. Twelve out of 19 patients with midline shift died .Out of the 7 live patients 1 was in vegetative state ( category4),5 were severely disabled ( category-3)and one patient showed good recovery. p value was 0.006 ( significant). That means midline shift was associated with poor outcome.

Subarachnoid extension of bleed was noted in 20 (i.e.26\%)of cases of IPH. Of this, sixteen patients died (category-5), two were in category-3, one was in category-2 and one showed good recovery( category1). $\mathrm{p}$ value was 000( significant). That means subarachnoid extension of bleed was associated with poor outcome.Based on Glasgow coma scale patients were classified into two groups i.e. GCS $\leq 8$ and more than 8. Out of 24 patients who had GCS $\leq 8$, eighteen patients died and six were alive. Out of 52 patients who had 
GCS $>8$, only 10 patients died, and 42 were alive. $\mathrm{P}$ value $<.000$ (highly significant). We have classified the patients with intraparenchymal hemorrhage according to location i.e. whether infratentorial or supra tentorial .Out of fourteen patients with infratentorial bleed 6(43\%) died and 8(57\%) were alive. Out of 8 live patients one was severely disabled ( category-3), five were in category-2, two patients showed good recovery( category-1). p value was 0.605 (not significant). There was no significant relation between location of bleed and the outcome.The significant predictors of 30 day mortality and morbidity in our study were intraventricular hemorrhage, midline shift, sub arachnoid extension of bleed, Glasgow coma scale at admission and volume of hematoma $30 \mathrm{ml}$ and above. No definite association noted with location ie, infratentorial location of bleed. But out of 14 infratentorial bleeds 4 were brainstem bleed. Death rate was $100 \%$ in brainstem bleed in this study irrespective of volume or other parameters. This showed that instead of infratentorial location, brainstem probably might be an important predictor for 30 day mortality. According to RM Yousuf, the overall mortality rate was $32.5 \%$ The significant independent predictors of acute in- hospital mortality were Glasgow Coma Scale (GCS) on admission, posterior fossa bleed, hematoma volume $>60 \mathrm{ml}$, midline shift and intraventricular extension of hemorrhage . According to Helweg-Larsen S, the acute mortality of ICH was 27\%. Determinant for the immediate prognosis was the level of consciousness and the volume of the hematoma (crucial size was $50 \mathrm{ml}$ ). Intraventricular hemorrhage was a bad prognostic sign only in the ganglionic-thalamic hematomas. ${ }^{11}$

According to P. Daverat, the mortality rate during the first month following ICH was $31 \%$. The significant independent predictors of early (30-day) mortality were hemorrhage size, midline shift, and intraventricular spread of the hemorrhage ${ }^{12}$. Broderick et al. found that the mortality rate at one month was best predicted by determining the initial score on the Glasgow Coma Scale $($ less $<9)$ and the initial volume of the hematoma $(>60)^{13}$. According to Anderson CS et al, the overall 28 day case fatality was $35 \%$, but this varied from $100 \%$ for hemorrhages in the brainstem to $22 \%$ for those in the basal ganglionic or thalamic region. Other predictors of early death were intraventricular extension of blood, volume of hematoma, mass effect, and coma and severe paresis at onset ${ }^{14}$. According to Michel Lelo Tshikwela et al, 30-day mortality rate was $35 \%$. ICH volume $>25 \mathrm{ml}$, presence of coma, left hemispheric site of ICH were identified as significant and independent predictors of 30-day mortality. Midline shift $>7 \mathrm{~mm}$, a consequence of ICH volume, was also a significant predictor of mortality 15

Intra ventricular extension of bleed, hematoma size and GCS at admission is significant predictors of mortality in all above mentioned studies. In this study also there was good correlation between these factors and 30 day mortality. Midline shift was mentioned as significant predictor of mortality in some of above studies. This study also showed good correlation between midline shift and 30 day mortality.

In this study, sub arachnoid extension of bleed was found as a good predictor of mortality. According to Alma M. Buensuceso, the only strong predictor of mortality on CT scan findings is the presence of subarachnoid hemorrhage, having three times higher risk of dying compared to those patients without $\mathrm{SAH}^{16}$. No significant correlation was found between infra tentorial location of bleed and mortality in this study, but mortality was $100 \%$ in brainstem hemorrhage here .

According to Anderson CS et al also there was good correlation between brainstem hemorrhage and 30 day mortality.

\section{Conclusions}

MDCT provides an excellent imaging modality for early detection of intracranial hemorrhage which helps to improve patient management. In the present study, an analysis of 100 patients with spontaneous intracranial hemorrhage was done. This study concluded the following aspects of intracranial hemorrhage:

- Incidence of non traumatic spontaneous intracranial hemorrhage was high around 60 years of age. Age distribution for IPH was maximum between 60-69 age groups. Age distribution for SAH was maximum between 50-59 age groups.

- Sex incidence of intracranial hemorrhage was high in male. But in SAH the incidence was more in females.

- Intraparenchymal hemorrhage was the commonest variety of non traumatic spontaneous intracranial hemorrhage .i.e. more than thrice of subarachnoid hemorrhage.

- Basal ganglia was the commonest location of intraparenchymal hemorrhage.

- Hypertension was the major causative factor for non-traumatic spontaneous intracranial hemorrhage.

- Among the patients with intraparenchymal hemorrhage, about half of them showed satisfactory outcome and one third patients died.

- The significant predictors of 30 days mortality were intraventricular hemorrhage, midline shift, sub arachnoid extension of bleed, low GCS on admission $(\leq 8)$ and volume of hematoma $30 \mathrm{ml}$ and above.

\section{References}

[1]. Robert R Edelman, John Hesselink, Michael Zlatkin. Clinical Magnetic Resonance Imaging ; brain intracranial hemorrhage, Third edition 2005, Saunders; 1287-1288.

[2]. Adnan I QureshI, Stanley Tuhri ,Joseph P Broderick, H Hunt Batjer, Hideki 
[3]. Hondo, Danielf Hanley. Spontaneous intracerebral hemorrhage. N Engl J Med 2001; 344( 19):1450-1460

[4]. Feigin VL, Rinkel GJ, Lawes CM, et al. Risk factors for subarachnoid hemorrhage: an updated systematic review of epidemiological studies. Stroke 2005; 36 (12): 2773-80.

[5]. Justine Elliott, Martin Smith. The Acute Management of Intracerebral Hemorrhage; A Clinical Review. Review article Anesthesia \& Analgesia Anesth Analg; 2010; 110:1419 - 27.

[6]. Azra Zafar, Farrukh Shohab Khan .Clinical and radiological features of intracerebral hemorrhage in hypertensive. Department of Neurology, Liaquat National JPMA 2008

[7]. Kase CS, Williams JP, Wyatt DA et al. Lobar Intracerebral hemorrhage, clinical and computed tomography analysis of 22 cases. Neurology 1982; 32: 1146-1150.

[8]. RM Yousuf, ARM Fauzi , AR Jamalludin, et.al. Predictors of in-hospital mortality in primary intracerebral hemorrhage in East Coast of Peninsular Malaysia. Neurology Asia 2012; 17(2) : 93 - 99

[9]. Lt Col MN Swamy. Management of Spontaneous Intracerebral Haemorrhage. MJAFI 2007; $63: 346-349$.

[10]. Douglas MA, Haerer AF. Long-term prognosis of hypertensive intracerebral hemorrhage. Stroke 1982;13:488-491.

[11]. Dixon AA, Holness RO, Howes WJ, Garner JB. Spontaneous intracerebral hemorrhage: An analysis of factors affecting prognosis. Can J Neurol Sci 1985;12:267-27.

[12]. Helweg Larsen S, Sommer W, Strange P, Lester J, Boysen G. Prognosis for patients treated conservatively for spontaneous intracerebral hematomas. Stroke 1984;15:1045-1048

[13]. P Daverat, J P Castel, J F Dartigues, J M Orgogozo. Death and Functional Outcome After Spontaneous Intracerebral Hemorrhage Prospective Study of 166 Cases Using Multivariate Analysis. Stroke 1991;22:1-6

[14]. Broderick JP, Brott TG, Duldner JE, Tomsick T, Huster G. Volume of intracerebral hemorrhage: a powerful and easy-to-use predictor of 30-daY mortality Stroke 1993;24:987-93.

[15]. Anderson CS, Chakera TM, Stewart-Wynne EG, Jamrozik KD . Spectrum of primary intracerebral haemorrhage in Perth, Western Australia, 1989-90: incidence and outcome. J Neurol Neurosurg Psychiatry 1994; 57(8):936.

[16]. Michel Lelo Tshikwela, Benjamin Longo-Mbenza. Spontaneous intracerebral haemorrhage:clinical and computed tomography findings in predictiing in hospital mortality in Central Africans. J Neurosci Rural Pract. 2012; 3 (2):115-20

[17]. Alma M Buensuceso . Predictors of Mortality Based on CT scan Findings of Patient Admitted Due to Hypertensive Intracerebral Hemorrhage at the Philippine Heart Center. Phil Heart Center J 2007; 13(2):155-160. 